\title{
ANSYS-Based Birefringence Property Analysis of Side-Hole Fiber Induced by Pressure and Temperature
}

\author{
Xinbang $\mathrm{ZHOU}^{1}$ and Zhenfeng GONG ${ }^{2 *}$ \\ ${ }^{1}$ School of Ocean Science and Technology, Dalian University of Technology. Panjin, Panjin, 124000, China \\ ${ }^{2}$ College of Physics and Optoelectronics Engineering, Dalian University of Technology, Dalian, 116024, China \\ *Corresponding author: Zhenfeng GONGＥ-mail: 342062580@mail.dlut.edu.cn
}

\begin{abstract}
In this paper, we theoretically investigate the influences of pressure and temperature on the birefringence property of side-hole fibers with different shapes of holes using the finite element analysis method. A physical mechanism of the birefringence of the side-hole fiber is discussed with the presence of different external pressures and temperatures. The strain field distribution and birefringence values of circular-core, rectangular-core, and triangular-core side-hole fibers are presented. Our analysis shows the triangular-core side-hole fiber has low temperature sensitivity which weakens the cross sensitivity of temperature and strain. Additionally, an optimized structure design of the side-hole fiber is presented which can be used for the sensing application.
\end{abstract}

Keywords: Fiber optics; birefringence; pressure measurement; temperature

Citation: Xinbang ZHOU and Zhenfeng GONG, "ANSYS-Based Birefringence Property Analysis of Side-Hole Fiber Induced by Pressure and Temperature," Photonic Sensors, 2018, 8(1): 13-21.

\section{Introduction}

Elasto-optic and thermo-optic effects exist in all kinds of optical fibers, and both of them affect the birefringence property of the fiber under different pressures and temperatures. Nowadays, side-hole fiber has been widely used to pressure sensing [1-6] because of its unique property such as high pressure sensitivity, low temperature sensitivity, and good temperature compensation capability [7]. However, there is no analysis about the optimized structure of the side-hole fibers. Most of these studies are confined to the side-hole fibers with circular holes, and the problem of cross sensitivity between the temperature and strain still exists. In this paper, we propose two novel side-hole fibers with rectangular holes and triangular holes. We analyze the birefringence property of the side-hole fibers under different pressures and temperatures. Comparing the influences of the shape, size, and position of the holes on the birefringence property of these three kinds of side-hole fibers, we demonstrate that the side-hole fiber with triangular holes is more suitable for strain sensing, because the triangular-core side-hole fiber has a lower temperature sensitivity, which solves the problem of cross sensitivity of temperature and strain very well.

\section{Principle of birefringence}

The birefringence property of the optical fiber mainly includes two aspects, which are inherent birefringence and induced birefringence. Inherent birefringence is formed during the fiber fabrication process. Once the optical fiber is fabricated, it is

Received: 5 June 2017 / Revised: 15 December 2017

(C) The Author(s) 2017. This article is published with open access at Springerlink.com

DOI: $10.1007 / \mathrm{s} 13320-017-0434-0$

Article type: Regular 
difficult to change the inherent birefringence. The induced birefringence is caused by the change in external environment conditions. An anisotropic stress distribution is induced in the core of the fiber, which can further generate fiber birefringence via the photo-elastic effect. As a result, we can use the induced birefringence of the optical fiber to measure the external conditions such as strain, temperature, and refractive index.

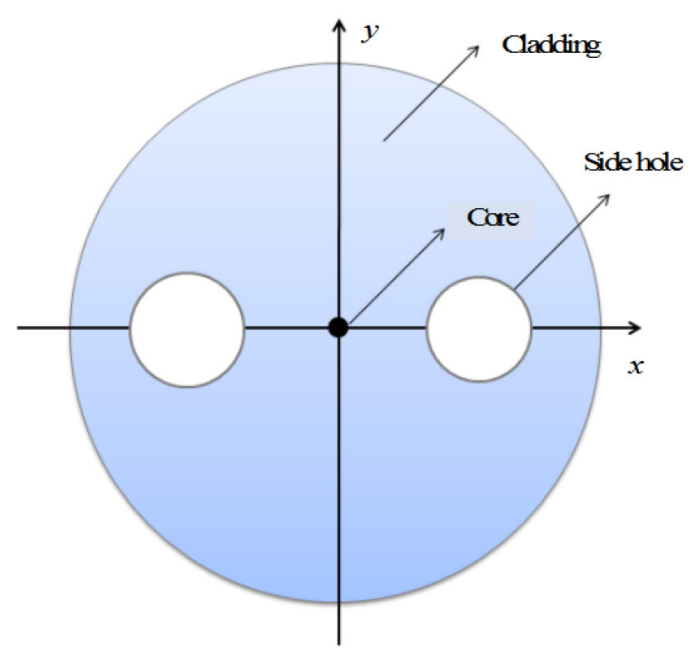

Fig. 1 Cross-section schematic of circular-core side-hole fibers.

A cross-section view of a common circular-core side-hole fiber is shown in Fig. 1. The radius of the fiber is $62.5 \mu \mathrm{m}$. According to the theory of the elasto-optic effect [8], the refractive indexes of the fiber core in the $x$ and $y$ directions can be expressed as [9]

$$
\begin{aligned}
& n_{x}=n_{x 0}+C_{1} \delta_{x}+C_{2} \delta_{y} \\
& n_{y}=n_{y 0}+C_{2} \delta_{x}+C_{1} \delta_{y}
\end{aligned}
$$

where $n_{x 0}$ and $n_{y 0}$ are the effective refractive indexes in the $x$ and $y$ directions of the core without ambient pressure, respectively, $C_{1}$ and $C_{2}$ refer to stress elasto-optic coefficients of the fiber, and $\delta_{x}$ and $\delta_{y}$ present the strains of the core in the $x$ and $y$ directions, respectively. The birefringence of the fiber is defined as follows [10]:

$$
B=n_{x}-n_{y}=\left(n_{x 0}-n_{y 0}\right)+\left(C_{1}-C_{2}\right)\left(\delta_{x}-\delta_{y}\right) .
$$

From (3), we know that the relationship between the birefringence of the fiber and the strain difference in the $x$ and $y$ directions is linear. In other words, the birefringence property analysis of the optical fiber can be converted into the strain difference analysis of the core of the fiber.

\section{Simulation results of ANSYS}

To numerically illustrate the influences of different pressures and temperatures on the birefringence property of side-hole fibers, we use the finite element analysis method [11] namely ANSYS to simulate the strain field distribution over the cross section of the fiber at a given pressure or temperature.

\subsection{Effect of strain}

In this simulation, the side-hole fibers are in the liquid environment of $20^{\circ} \mathrm{C}$, and the pressure of $1 \times 10^{5} \mathrm{~Pa}$ acts uniformly on the fiber surface. The birefringence properties of circular-hole, rectangular-hole, and triangular-hole side-hole fibers are compared. Through this simulation results, we get the optimal size, shape, and location of the holes of side-hole fiber, which exhibits a high strain sensitivity and a low temperature sensitivity.

\subsubsection{Circular holes}

For the side-hole fiber with circular holes, we hope to find the suitable radius and position of the holes in order to realize the largest strain sensitivity. We define $\delta$ as the strain difference between $y$-axis and $x$-axis of the core, which can be expressed as follows:

$$
\delta=\delta_{y}-\delta_{x} .
$$

Firstly, we fix the position of the holes and change the radius from small to large, then we obtain a relationship between the strain difference and the radiuses of the holes; secondly, we keep the radius of the holes constant and increase the distance between the core and the holes. As a result, we achieve a relationship between the strain difference and positions of the holes. 


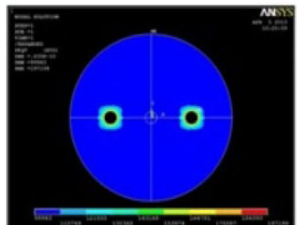

(1) $r=5 \mu \mathrm{m}$

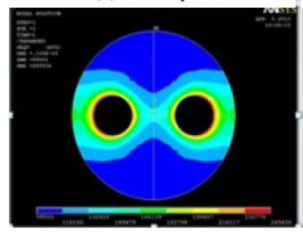

(3) $r=15 \mu \mathrm{m}$

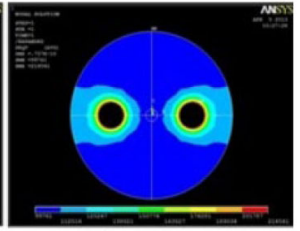

(2) $r=10 \mu \mathrm{m}$

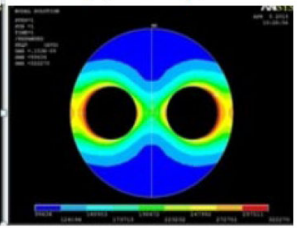

(4) $r=20 \mu \mathrm{m}$

(a)

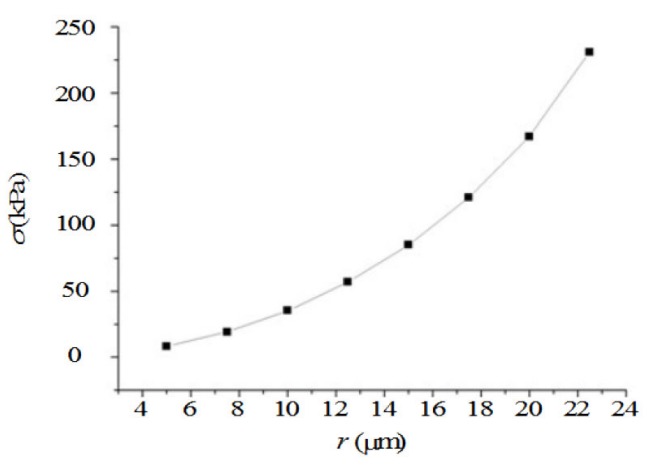

(b)

Fig. 2 Strain distributions of the circular-core side-hole fiber: (a) strain field distribution of different side-hole fibers (the radii of the holes are $5 \mu \mathrm{m}, 10 \mu \mathrm{m}, 15 \mu \mathrm{m}$, and $20 \mu \mathrm{m}$, respectively. The length between the centers of the core and holes is fixed at $31.25 \mu \mathrm{m}$ ) and (b) strain difference changes with radii of the side holes.

The strain distributions of the circular-core side-hole fiber with different side holes are shown in Fig. 2(a). Different intensities correspond to different amplitude levels of the strain field. We obtain a relationship between the strain difference of the core and the size of radius from Fig. 2(a), which is illustrated in Fig. 2(b). We conclude that the strain difference increases gradually as the radii. According to (3), it means that the birefringence property of the fiber is further generated when the radius of the holes becomes big. Therefore, the side-hole fiber with larger holes is more sensitive for pressure sensing.

The length between the centers of the core holes in Fig. 3(a) increases from $20 \mu \mathrm{m}$ to $45 \mu \mathrm{m}$. The radius of the holes is fixed at $10 \mu \mathrm{m}$. From Fig.3(a), we obtain the strain difference of the core when the holes are at different positions. The relationship between the strain difference and the length from center of the core to center of the holes is displayed in Fig. 3(b). The strain difference of $y$-axis and $x$-axis of the core decreases gradually when the holes move to the edge of the fiber.
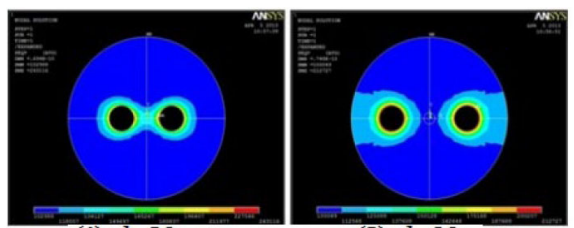

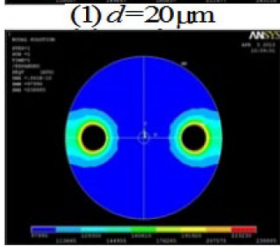

(3) $d=40 \mu \mathrm{m}$

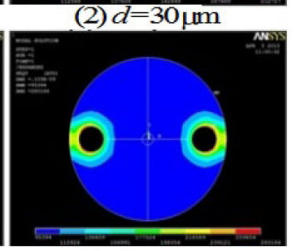

(4) $d=45 \mu \mathrm{m}$ (a)

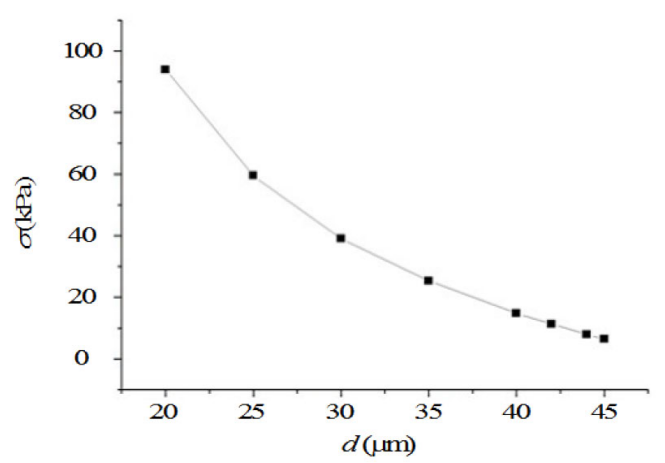

(b)

Fig. 3 Strain distributions of the circular-core side-hole fiber: (a) the strain field distribution of different side-hole fibers with fixed holes radius of $10 \mu \mathrm{m}$ and the lengths between the core and holes corresponding to $20 \mu \mathrm{m}, 30 \mu \mathrm{m}, 40 \mu \mathrm{m}$, and $45 \mu \mathrm{m}$, respectively) and (b) strain difference versus length between the centers of the core and holes.

It can be deduced from above results that $\delta_{x}$ and $\delta_{y}$ significantly change with the radius and position of the holes, meanwhile, $\delta_{x}$ and $\delta_{y}$ are both positive. Compared with the single mode fiber, due to the existence of side holes, the difference between $\delta_{x}$ and $\delta_{y}$ increases, which generates stronger birefringence property. Furthermore, the existence of side holes has a great impact on 
the strain distribution of the fiber. Because $\delta_{y}$ has a larger variation than $\delta_{x}$ under the same condition, the $x$-axis is the fast axis of the fiber.

\subsubsection{Rectangular holes}

In order to find the optimized structure of the side holes, we propose two novel side-hole fibers. Figure 4 exhibits the cross-section drawing of the rectangular-core side-hole fiber.

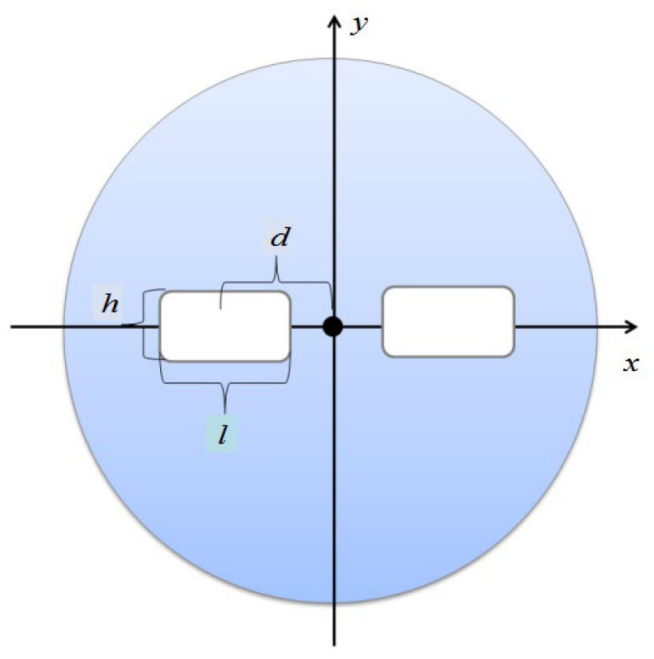

Fig. 4 Cross-section schematic of the rectangular-core side-hole fiber.

Figures 5(a) to 5(c) show the strain distributions of different rectangular-core side-hole fibers. The lengths $l$ in Fig. 5(a) are $10 \mu \mathrm{m}, 20 \mu \mathrm{m}, 30 \mu \mathrm{m}$, and $40 \mu \mathrm{m}$, respectively. The height $h$ and distance $d$ are fixed at $20 \mu \mathrm{m}$ and $31.25 \mu \mathrm{m}$, respectively. Figure 5(b) shows the change in the height $h$ of the holes. The heights are $20 \mu \mathrm{m}, 40 \mu \mathrm{m}, 60 \mu \mathrm{m}$, and $75 \mu \mathrm{m}$, respectively. The length $l$ and distance $d$ are fixed at $20 \mu \mathrm{m}$ and $31.25 \mu \mathrm{m}$, respectively. The length $d$ between the centers of the core and holes in Fig. 5(c) increases from $10 \mu \mathrm{m}$ to $38 \mu \mathrm{m}$, and the length $l$ and height $h$ are all fixed at $20 \mu \mathrm{m}$. Figure 5(d) shows that the strain difference can be tuned by varying the length $l$ of the rectangular holes. As the length $l$ increases, the strain difference increases gradually. In Fig. 5(e), the relationship between the strain difference and height $h$ is plotted. As height $h$ increases, the strain difference has the largest value at about $47 \mu \mathrm{m}$. Figure 5(f) depicts the effect of distance $d$ on the strain difference, which has an inverse proportional relationship.

\subsubsection{Triangular holes}

For the side-hole fiber with equilateral triangle holes, the largest strain sensitivity can be achieved by seeking the suitable size $a$ and length $d$. Figure 6 exhibits the cross-section drawing of the triangular-core side-hole fiber.

Figures 7(a) and 7(b) show the strain distribution of different triangular-core side-hole fibers. The lengths $a$ in Fig. 7(a) are $5 \mu \mathrm{m}, 10 \mu \mathrm{m}, 15 \mu \mathrm{m}$, and $20 \mu \mathrm{m}$, respectively, with the distance $d$ fixed at $31.25 \mu \mathrm{m}$. Figure 7(b) shows that the distance $d$ is $15 \mu \mathrm{m}, 25 \mu \mathrm{m}, 35 \mu \mathrm{m}$, and $45 \mu \mathrm{m}$, respectively, with the length $a$ fixed at $10 \mu \mathrm{m}$. Figure 7(c) shows that as the length $a$ increases, the strain difference increases gradually. In Fig. 7(d), the relationship between the strain difference and distance $d$ is plotted. As distance $d$ increases, the strain difference decreases gradually.

From the simulated results of three kinds of side-hole fibers, we find that the strain sensitivity increases with the overall size of the holes and decreases with the distance between the centers of the core and holes. Comparing these three kinds of side-hole fibers, the side-hole fiber with rectangular holes is more suitable for pressure sensing without considering the impact of temperature shown in Fig. 5(d). One possible reason is that compared with the circle holes, the outline of rectangle holes is less smooth, and the strain distribution in the rectangular-core side-hole fiber is more uneven, which might lead to the bigger strain difference in the core of the fiber. Compared with the triangle holes, the overall size of rectangle holes is bigger, so its strain difference is bigger. The conclusion is consistent with that in [12]. 


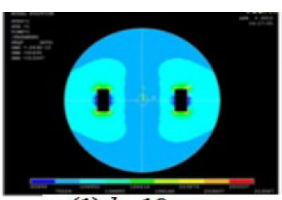

(1) $l=10 \mu \mathrm{m}$

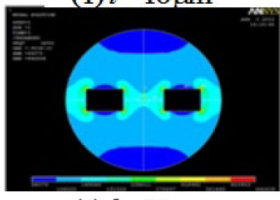

(3) $l=30 \mu \mathrm{m}$

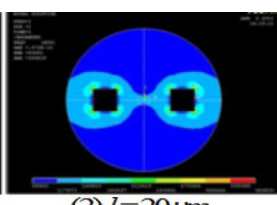

(2) $l=20 \mu \mathrm{m}$

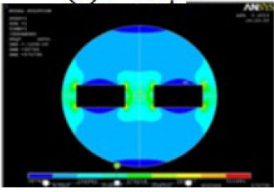

(4) $l=40 \mu \mathrm{m}$

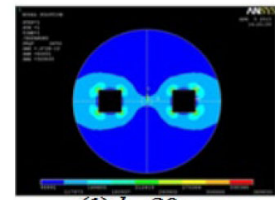

(1) $h=20 \mu \mathrm{m}$

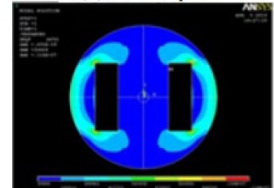

(3) $l=60 \mu \mathrm{m}$
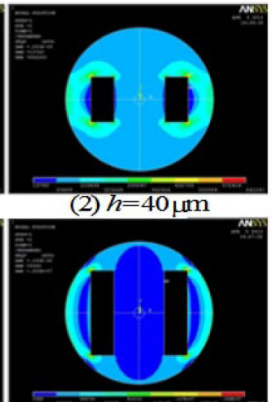

(4) $l=75 \mu \mathrm{m}$

(a)
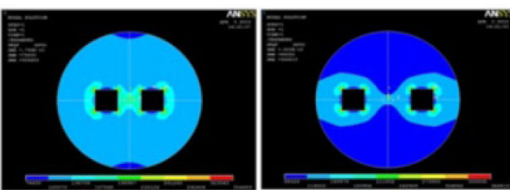

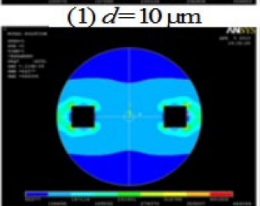

(3) $d=30 \mu \mathrm{m}$

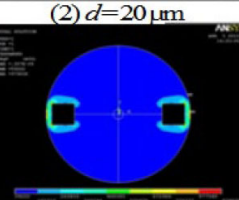

(4) $d=38 \mu \mathrm{m}$

(c)

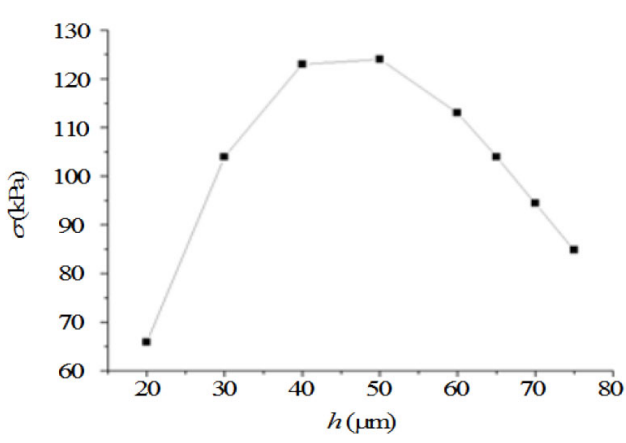

(e)

(b)

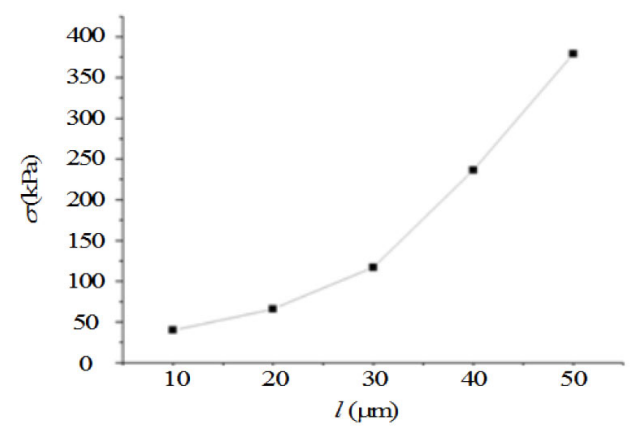

(d)

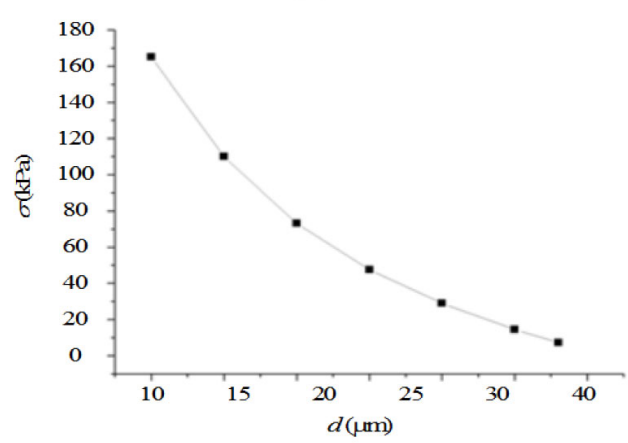

(f)

Fig. 5 Strain field distribution of different rectangular-core side-hole fibers: (a) varying length $l$ of rectangular holes with fixed $h=$ $20 \mu \mathrm{m}$ and $d=31.25 \mu \mathrm{m}$, (b) varying height $h$ of rectangular holes with fixed $l=20 \mu \mathrm{m}$ and $d=31.25 \mu \mathrm{m}$, (c) varying distance $d$ with fixed $l=20 \mu \mathrm{m}$ and $h=20 \mu \mathrm{m}$, strain difference versus (d) length $l$, (e) height $h$ of rectangular holes, and (f) distance $d$ between the centers of the core and holes.

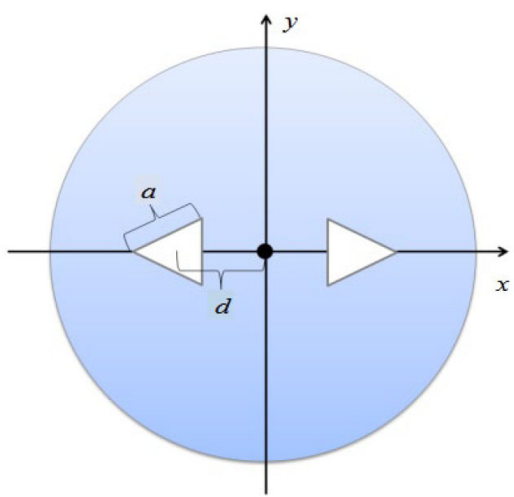

Fig. 6 Cross-section schematic of the triangular-core side-hole fiber. 


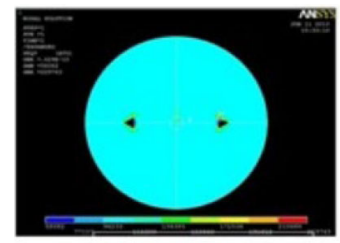

(1) $a=5 \mu \mathrm{m}$

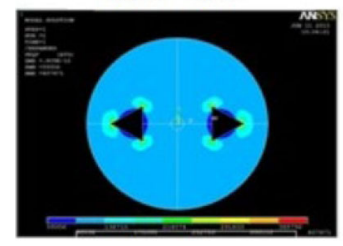

(3) $a=15 \mu \mathrm{m}$

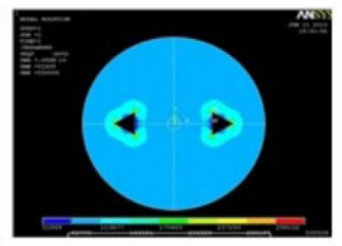

(2) $a=10 \mu \mathrm{m}$

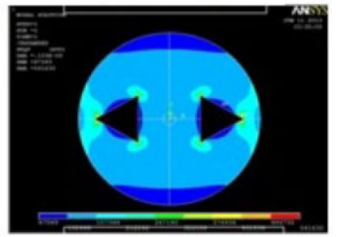

(4) $a=20 \mu \mathrm{m}$

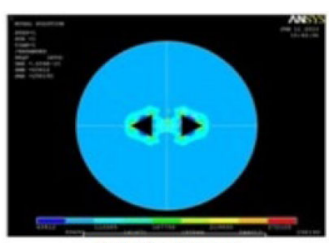

(1) $d=15 \mu \mathrm{m}$

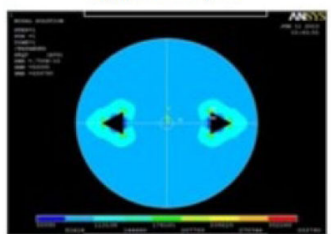

(3) $d=35 \mu \mathrm{m}$

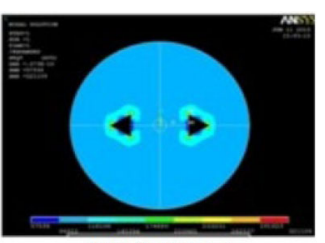

(2) $d=25 \mu \mathrm{m}$

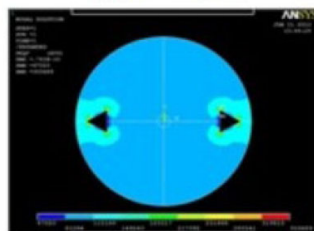

(4) $d=45 \mu \mathrm{m}$ (a)

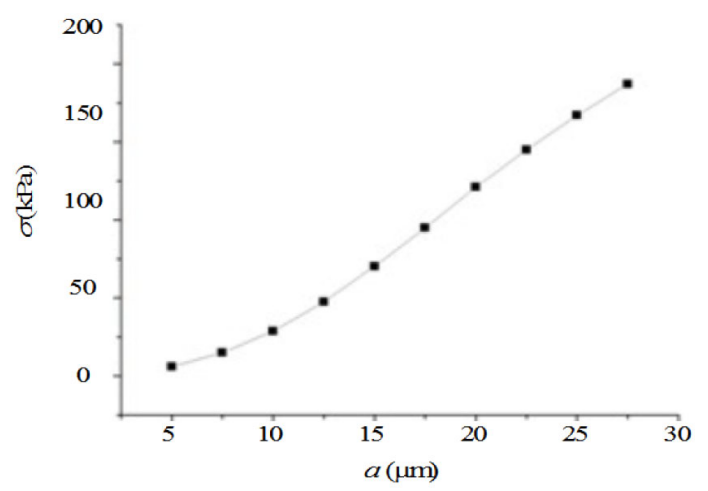

(c) (b)

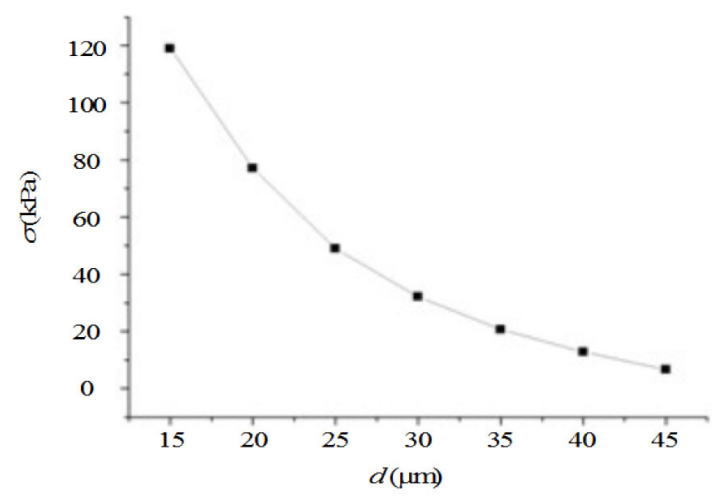

(d)

Fig. 7 Strain field distribution of different triangular-core side-hole fibers: (a) varying length $a$ of triangular holes with fixed $d=$ $31.25 \mu \mathrm{m}$, (b) varying distance $d$ with fixed $a=10 \mu \mathrm{m}$, strain difference versus (c) length $a$ and (d) distance $d$.

\subsection{Effect of temperature}

The temperature can also influence the birefringence property of the fiber. Assuming no external pressure acts on the surface of the fiber, and the temperature is $80^{\circ} \mathrm{C}$. The strain differences of different side-hole fibers are simulated.

\subsubsection{Circular holes}

Figure 8(a) shows the influence of the radius of the holes on the strain difference at the temperature of $80^{\circ} \mathrm{C}$. The strain difference increases gradually with the radius $r$. As shown in Fig. 8(b), if the distance between the centers of the core and holes decreases, the birefringence effect of the core becomes more obvious.

As we know, physical mechanisms of the birefringence of the fiber induced by the pressure and temperature are elasto-optic effect and thermo-optic effect, respectively. Comparing Fig. 2(b) with Fig. 8(a) and Fig. 3(b) with Fig. 8(b), their overall trends are nearly consistent.

\subsubsection{Rectangular holes}

As shown in Fig. 9, for the rectangular-core side-hole fiber, the birefringence effect is obvious when the length of the rectangular holes is large and the edge of the holes is near from the core.

\subsubsection{Triangular holes}

As shown in Fig. 10, for the triangular-core side-hole fiber, the strain difference increases with the size of the holes $a$ and decreases with the distance $d$. From the above results, we find that the 
temperature sensitivities of all of these three kinds of side-hole fibers increase with the size of the holes

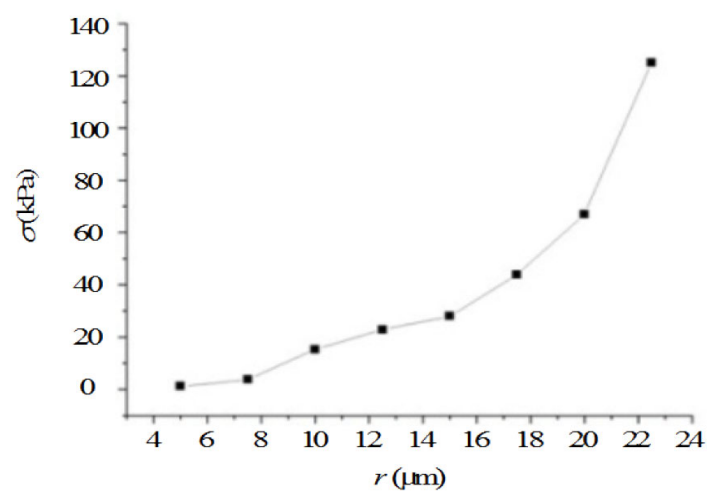

(a) and decrease with the distance between the core and the holes.

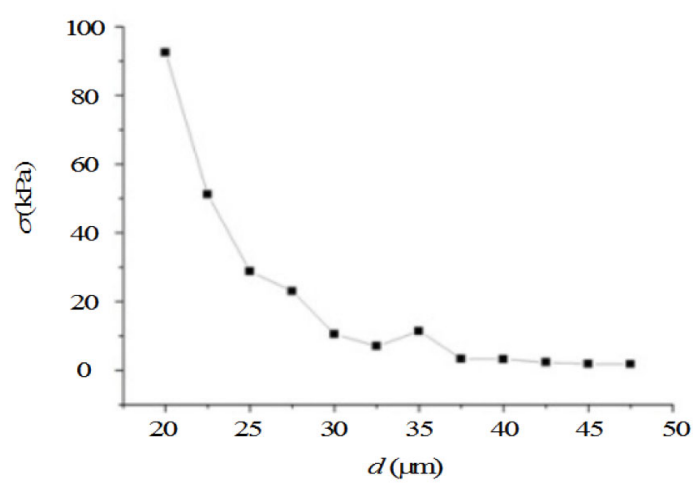

(b)

Fig. 8 Strain difference changes with (a) radius $r$ and (b) distance $d$ at the temperature of $80^{\circ} \mathrm{C}$.

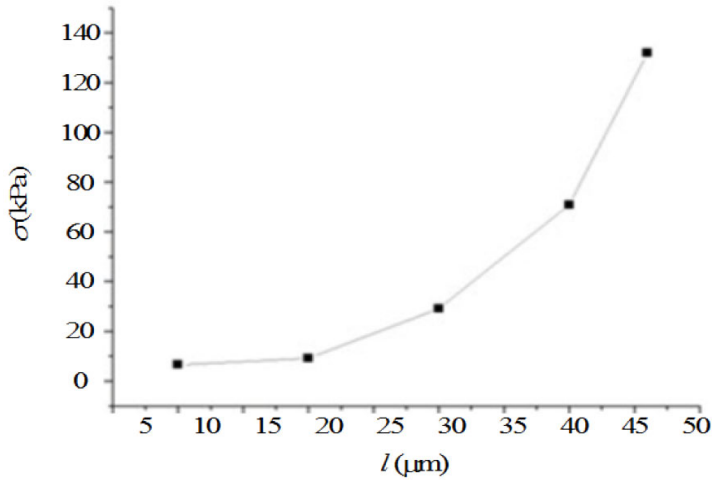

(a)

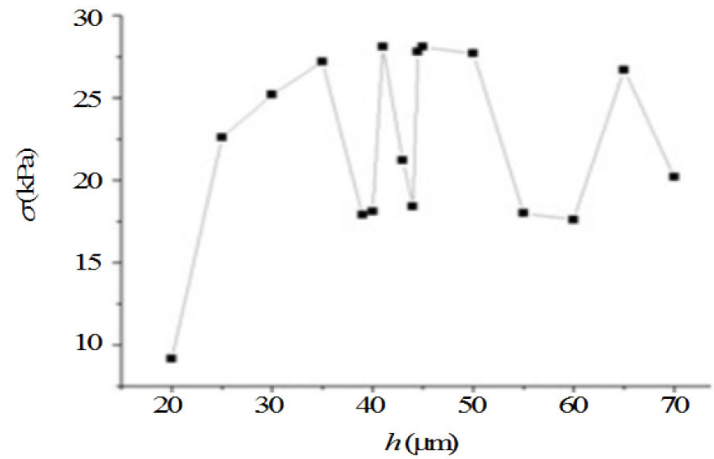

(b)

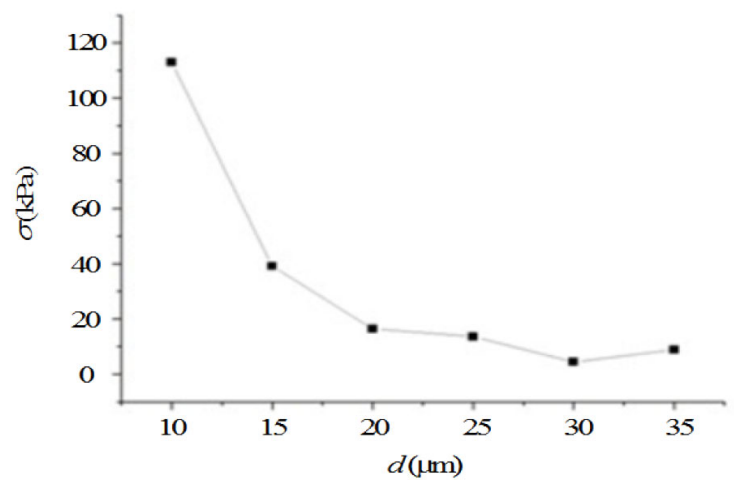

(c)

Fig. 9 Strain difference changes versus (a) length $l$ (height $h=20 \mu \mathrm{m}$ and distance $d=31.25 \mu \mathrm{m})$, (b) height $h(l=20 \mu \mathrm{m}$ and $d=$ $31.25 \mu \mathrm{m})$ of rectangular holes, and (c) distance $d(l=20 \mu \mathrm{m}$ and $h=20 \mu \mathrm{m})$ between the centers of the core and holes at the temperature of $80^{\circ} \mathrm{C}$. 


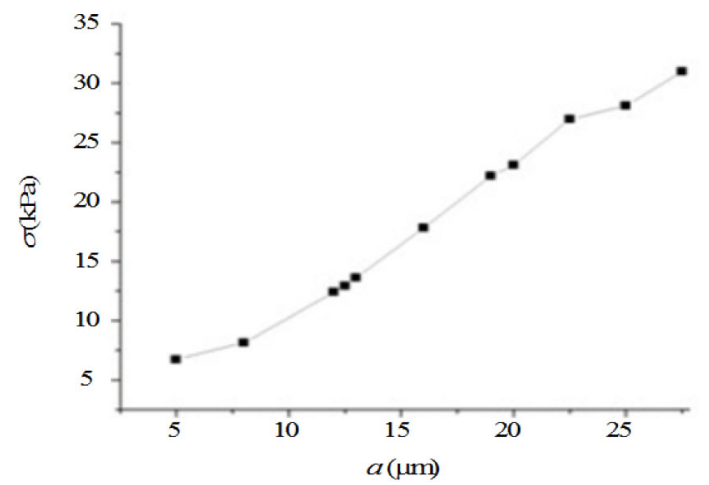

(a)

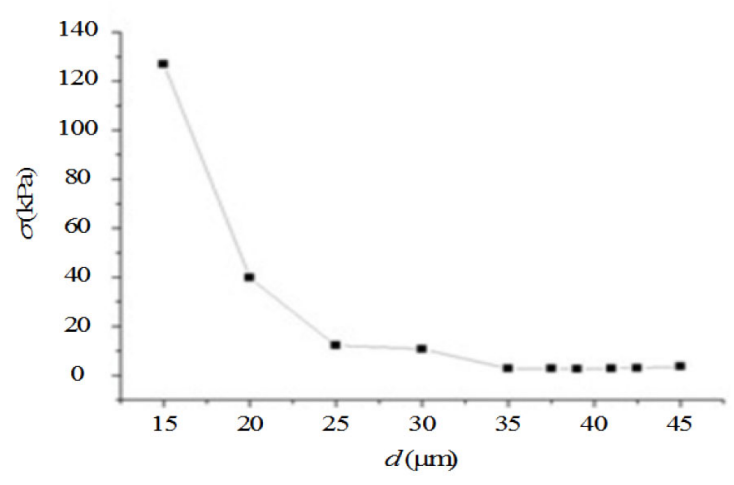

(b)

Fig. 10 Strain difference changes with (a) length $a$ and (b) distance $d$ at the temperature of $80^{\circ} \mathrm{C}$.

It is assumed that the sizes of the holes in these three kinds of side-hole fibers are comparable, and the changes in the strain differences of these three kinds of side-hole fibers are graphed as shown in Fig. 11. Figure 11(a) presents how the strain differences change with the size of holes of three kinds of side-hole fibers at the pressure of $1 \times 10^{5} \mathrm{~Pa}$ on the fiber surface, regardless of the influence by the temperature. It is known that the strain differences increase with the size of the holes. The strain differences versus the sizes of the holes at the temperature of $80^{\circ} \mathrm{C}$ with no pressure are shown in Fig. 11(b). We find that when the size of the holes increases gradually, the strain difference of the triangular-core side-hole fiber has a slow growth. It means using triangular-core side-hole fibers for strain sensing, the strain sensitivity can be improved by enlarging the size of the holes, meanwhile, the temperature sensitivity is controlled in a small level. Therefore, the problem of the cross sensitivity between the temperature and strain is solved better.

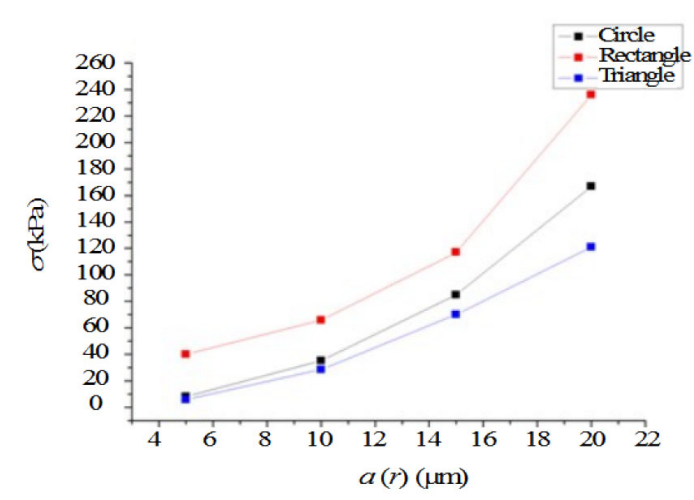

(a)

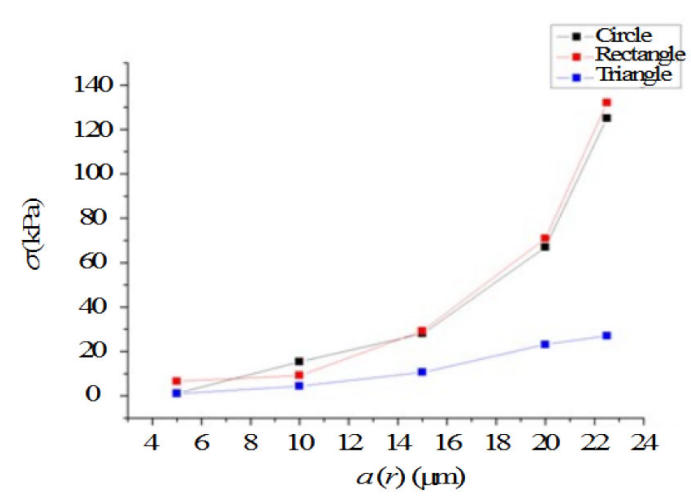

(b)

Fig. 11 Changes in the strain differences of these three kinds of side-hole fibers: (a) strain differences versus holes size $a(r)$ of three kinds of side-hole fibers at the pressure of $1 \times 10^{5} \mathrm{~Pa}$ on the fiber surface and (b) strain differences versus with holes size $a(r)$ of three kinds of side-hole fibers at the temperature of $80^{\circ} \mathrm{C}$.

\section{Conclusions}

In summary, we analyze the influences of the shape, size, and position of the holes on the birefringence property of side-hole fibers under different pressures and temperatures. We use the finite element analysis method of ANSYS to simulate the strain field distribution over the cross section of the fiber at a given pressure of $1 \times 105 \mathrm{~Pa}$ and a given temperature of $80{ }^{\circ} \mathrm{C}$. The strain differences caused by the strain and temperature 
both increase with the size of the holes and decrease with the distance between the core and holes. The side-hole fiber with triangular holes has a much lower birefringence property if enlarging the size of the holes in order to get a big strain sensitivity, in other words, it may have a high strain sensitivity and a low temperature sensitivity at the same time. Therefore, the triangular-core side-hole fiber is more suitable than the circular-core and rectangular-core side-hole fibers for pressure sensing because it solves the problem of the cross sensitivity of temperature and strain very well.

\section{Acknowledgment}

This research is supported by the Fundamental Research Funds for the Central Universities [DUT 15RC(3) 112].

Open Access This article is distributed under the terms of the Creative Commons Attribution 4.0 International License (http://creativecommons.org/ licenses/by/4.0/), which permits unrestricted use, distribution, and reproduction in any medium, provided you give appropriate credit to the original author(s) and the source, provide a link to the Creative Commons license, and indicate if changes were made.

\section{References}

[1] D. M. Li, W. Zhang, and G. Y. Zhou, "Numerical analysis of a side-hole birefringent photonic crystal fiber with high-pressure sensitivity," Optical Engineering, 2016, 55(9): 097106-1-097106-6.

[2] E. R. Vera, C. M. B. Cordeiro, and P. Torres, "Highly sensitive temperature sensor using a Sagnac loop interferometer based on a side-hole photonic crystal fiber filled with metal," Applied Optics, 2017, 56(2):
$156-162$.

[3] A. Anuszkiewica, T. Martynkien, J. Olszewski, P. Mergo, and W. Urbanczyk, "Polarimetric sensitivity to hydrostatic pressure and temperature in a side-hole fiber with squeezed microstructure," Journal of Optics, 2015, 17(12): 125609-1-25609-6.

[4] T. Martynkien, G. Wojcik, P. Mergo, and W. Urbanczyk, "Highly birefringent polymer side-hole fiber for hydrostatic pressure sensing," Optics Letters, 2015, 40(13): 3033-3035.

[5] D. S. Moon, U. C. Paek, and Y. Chung, "Polarization controlled multi-wavelength Er-doped fiber laser using fiber Bragg grating written in few-mode side-hole fiber with an elliptical core," Optics Express, 2005, 13(14): 5574-5579.

[6] S. H. Lee, B. H. Kim, and W. T. Han, "Effect of filler metals on the temperature sensitivity of side-hole fiber," Optics Express, 2009, 17(12): 9712-9717.

[7] E. Chmielewska, W. Urbanczyk, and W. J. Bock, "Measurement of pressure and temperature sensitivities of a Bragg grating imprinted in a highly birefringent side-hole fiber," Applied Optics, 2003, 42(31): 6284-6291.

[8] Q. Zhang, N. Liu, T. Fink, H. Li, W. Peng, and M. Han, "Fibre-optic pressure sensor based on $\pi$-phase-shifted fiber Bragg grating on side-hole fiber," IEEE Photonics Technology Letters, 2012, 24(17): 1519-1522.

[9] Y. Liu, B. M. A. Rahman, and K. T. V. Grattan, "Thermal-stress-induced birefringence in bow-tie optical fibers," Applied Optics, 1994, 33(24): 5611-5616.

[10] H. M. Xie, P. Dabkiewicz, R. Ulrich, and K. Okamoto, "Side-hole fiber for fiber-optic pressure sensing," Optics Letters, 1986, 11(5): 333-335.

[11] T. J. R. Hughes, Finite element method: linear static and dynamic finite element analysis. America: Dover Publications, 2000: 1-704.

[12] Y. Wei, D. Y. Chang, K. Zheng, and S. S. Jian, "Research on the distribution of model electric field and birefringence in circular-core side-hole fibers," Journal of Optoelectronics Laser, 2007, 18(2): 154-158. 